\title{
Ermittlung eines kleinräumigen Brunneneinzugsgebiets un- ter Berücksichtigung hydrologischer und hydrogeologischer Unsicherheiten
}

\author{
Armin Matzl · Sebastian Handl · Ernest Mayr · Reinhard Perfler
}

Online publiziert: 23. Juli 2019

(C) Der/die Autor(en) 2019

Zusammenfassung Das südliche Wiener Becken stellt einen großen zusammenhängenden Aquifer dar, der vielerorts zur Gewinnung von Trinkwasser genutzt wird. Gleichzeitig werden große Teile landwirtschaftlich intensiv genutzt. Dadurch steigt der Druck auf das Grundwasser und für Wasserversorger das Bedürfnis nach einer ausreichenden Ausweisung von Schutzzonen in den Brunneneinzugsgebieten. Dafür ist eine möglichst genaue Kenntnis der Ausdehnung der Zuflussflächen zu den Brunnen entscheidend. In der gegenständlichen Untersuchung wurden die Zuflussflächen eines Brunnenfeldes am östlichen Rand des südlichen Wiener Beckens mithilfe eines numerischen Grundwassermodells ermittelt. Bei der modelltechnischen Umsetzung konnten hydrologische und hydrogeologische Parameter jedoch teilweise nur grob abgeschätzt werden. Aus diesem Grund wurden sechs Varianten ausgearbeitet und kalibriert. Durch die angewandte Methode bei der Kalibrierung konnten mehrere Parameterkombinationen gefunden werden, die eine ähnlich gute Modellanpassung liefern. Dadurch war es möglich, die Ergebnisse von insgesamt 302 Parametersätzen $\mathrm{zu}$ analysieren, um den Unsicherheiten und Kompromissen, die sich während der Modellerstellung zwangsläufig ergaben, mit einer größeren Anzahl an Berechnungen entgegenzuwirken. Die Ergebnisse stellen eine Bandbreite an möglichen Zuflussflächen dar, welche

DI A. Matzl, B.Sc. $(\bowtie) \cdot$ DI S. Handl • DI E. Mayr · PD DI Dr. R. Perfler Department

Wasser-Atmosphäre-Umwelt, Institut für Siedlungswasserbau, Industriewasserwirtschaft und Gewässerschutz, Universität für Bodenkultur Wien,

Muthgasse 18, 1190 Wien, Österreich armin.matzl@boku.ac.at anschließend statistisch ausgewertet wurden.

\section{Schlüsselwörter}

Grundwassermodellierung . Unmittelbarer Zustrombereich . Unsicherheitsbetrachtung · Monte Carlo

Determination of a small-scale well catchment area under consideration of hydrological and hydrogeological uncertainties

Abstract The southern Vienna basin is a huge aquifer which is often used for drinking water supply. At the same time, major parts are used for intensive farming. Due to this, the pressure on groundwater rises and water suppliers claim for larger protection areas around their wellfields. Therefore, the smallscale catchment area must be known and declared. Within this study, a numerical groundwater model was set up to determine the catchment area of a well field close to the eastern border of the southern Vienna basin. Uncertainties were discovered in respect of infiltration through the riverbed of the nearby river. Therefore six different variants of infiltration through the riverbed were developed and calibrated. Due to the applied method to calibrate the model, 302 different parameter combinations were found which lead to a similar good model fit. The catchment area was calculated for ten periods within each parameter combination. The result is a bandwidth of possible expansions of the small scale well field catchment area. In the end the results were evaluated statistically to get a more reliable result.

Keywords Groundwater modelling . Direct catchment area - Uncertainty consideration · Monte Carlo

\section{Einleitung}

Der Wasserleitungsverband Nördliches Burgenland betreibt nahe Neudörfl sein größtes Brunnenfeld mit einer bewilligten Entnahme von 6001/s aus zwei Brunnen. Derzeit kann das geförderte Grundwasser ohne Aufbereitung in das Versorgungsnetz eingespeist werden. Um die ausgezeichnete Qualität des Rohwassers beizubehalten, gilt es heute vorausschauend Maßnahmen zu setzen. Durch die Erstellung eines detaillierten numerischen Grundwassermodells rund um das Brunnenfeld wurden die 60-Tage-Zuflussflächen zu den Brunnen unter Berücksichtigung von hydrologischen und hydrogeologischen Unsicherheiten ermittelt.

\section{Untersuchungsgebiet}

Das Untersuchungsgebiet befindet sich südöstlich von Wiener Neustadt und umfasst das großräumige Einzugsgebiet des Brunnenfeldes Neudörfl. Die größten Teile des Modellgebiets liegen in Niederösterreich, das Brunnenfeld selbst befindet sich jedoch im Burgenland. Aufgrund der Größe des Grundwasserkörpers ist es rechentechnisch nicht vertretbar, diesen zur Gänze in der geforderten Genauigkeit in das Modell zu übernehmen. Gegen Osten wird er durch das Rosaliengebirge begrenzt, welches als Randbedingung zweiter Art im Modell implementiert wurde. An den übrigen Rändern des Modells wurden Wasserstände als Randbedingung erster Art zur Abgrenzung des Modellgebiets gegen den restlichen Grundwasserkörper herangezogen. Die Aquifermächtigkeit beträgt am östlichen Rand $0 \mathrm{~m}$ und reicht bis zu $90 \mathrm{~m}$ im Raum Wiener Neustadt. Die Lage des Grundwasserstauers wurde dem überregionalen Grundwassermodell von Kupfersberger et al. (2014) entnommen. Für die Beurteilung der Modellgüte standen 9 Grundwassermessstellen 


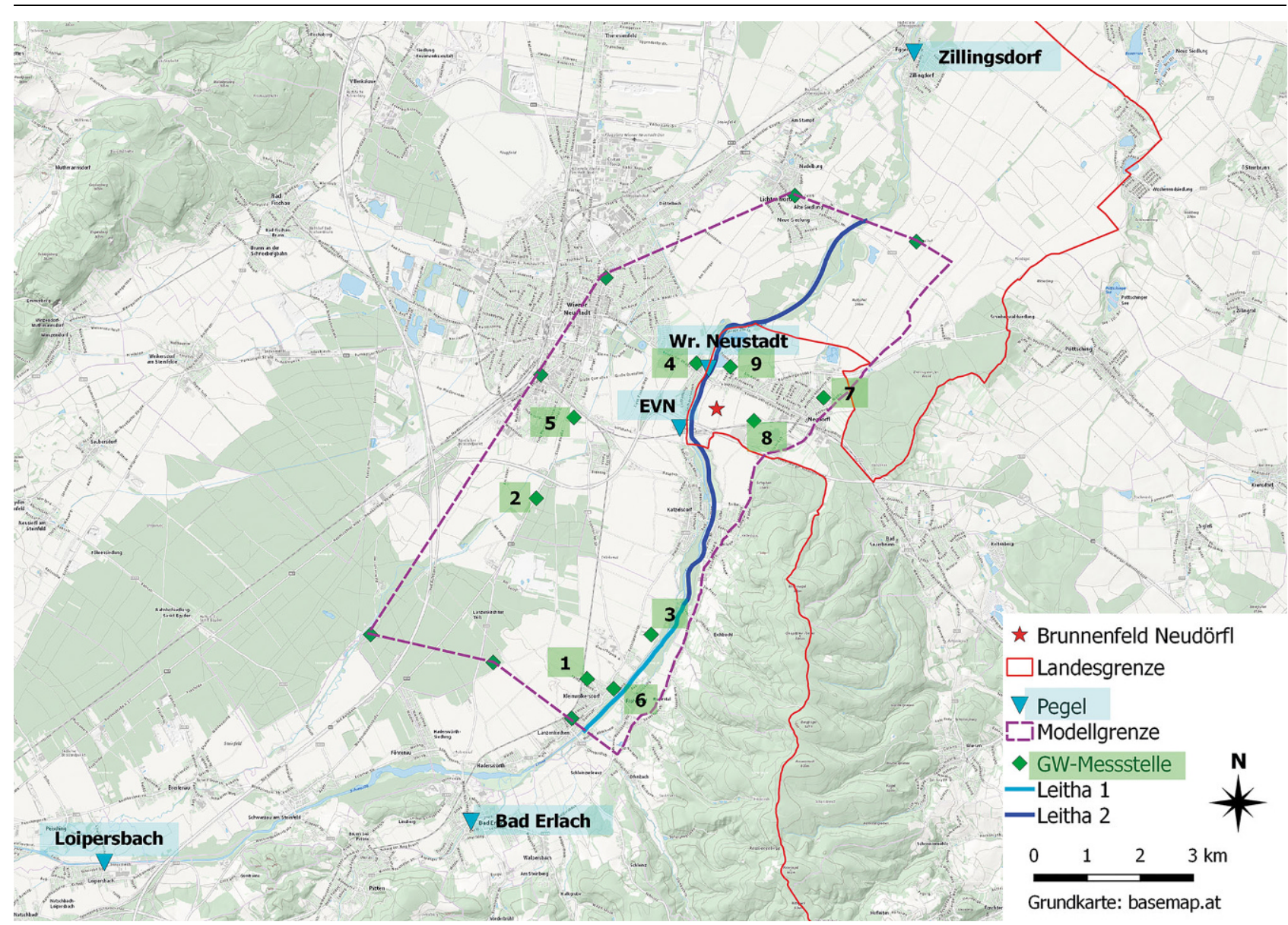

Abb. 1 Übersicht des Modellgebiets

im Untersuchungsgebiet zur Verfügung. Eine Übersicht zur Lage der Messstellen und Ausdehnung des Modellgebiets ist Abb. 1 zu entnehmen.

\subsection{Interaktion zwischen Grundwasser und Leitha}

Nachtnebel und Holzmann (2001) haben eine Studie zu den Abflussverhältnissen der Leitha bei Nieder- und Mittelwasserführung durchgeführt. Die Autoren unterstreichen in ihrer Untersuchung die Bedeutung der Infiltration in den Grundwasserkörper durch das Flussbett der Leitha. Deshalb wurde bei der Modellerstellung versucht, den Einfluss der Leitha auf den Wasserhaushalt des Grundwasserkörpers möglichst genau zu charakterisieren.

Bei Haderswörth fließt die Pitten in die Schwarza. Ab dort wird der Fluss Leitha genannt. Das Abflussregime ist stark anthropogen beeinflusst, da noch an der Schwarza der Kehrbach und beim Katzelsdorfer Rauwehr der Katzelsdorfer Werkskanal ausgeleitet wer- den. Diese beiden Kanäle sind künstlich geschaffen und es wird angenommen, dass deren Sohle dicht ausgeführt ist, um Wasserverluste für die anschließende energetische Nutzung zu reduzieren. Daher wurden die Werkskanäle für die folgenden Betrachtungen nicht berücksichtigt. Die untersuchten Flussabschnitte beziehen sich ausschließlich auf die Leitha.

Für die Bearbeitung standen Daten der Pegel Loipersbach, Bad Erlach, Wiener Neustadt (Straßenbrücke), Katzelsdorf (EVN) und Zillingsdorf der Jahre 1979-2017 zur Verfügung (Abb. 1). Für die Quantifizierung der Versickerung wurde die Leitha in zwei Bereiche unterteilt. Der erste reicht vom Pegel Loipersbach bis zum Katzelsdorfer Rauwehr und wird durch Gl. 1 beschrieben. Dabei wurden jeweils die Tagesmittelwerte der einzelnen Stationen herangezogen und subtrahiert. Der zweite Abschnitt beginnt beim Katzelsdorfer Rauwehr, endet beim Pegel Zillingsdorf und wird durch Gl. 2 beschrieben. Die Ergebnisse im Abschn. 1 für die Jahre
1979-2014 sind in Abb. 2 nach Monaten getrennt und in Form von Boxplots dargestellt. Daraus geht hervor, dass ganzjährig Wasser durch das Flussbett infiltriert.

Da das Modellgebiet nicht direkt bei einem Pegel beginnt beziehungsweise endet, musste abgeschätzt werden, wie viel Wasser auf den Fließstrecken außerhalb des Modells bereits versickert. Durch diese Schätzung ergaben sich Unsicherheiten. Um diesen $\mathrm{zu}$ entgegnen wurden insgesamt sechs Varianten ausgearbeitet. In Tab. 1 ist angeführt, welcher Anteil der Versickerung im Modellgebiet angesetzt wurde. In zwei Varianten wurde die Versickerung zusätzlich durch einen virtuellen Linearspeicher gedämpft.

$$
\begin{aligned}
\Delta V_{1}= & \left(Q_{\mathrm{EVN}}+Q_{W r \text { Neustadt }}\right) \\
& -\left(Q_{\text {Loipersbach }}+Q_{\text {Bad Erlach }}\right) \\
\Delta V_{2}= & Q_{\text {Zillingsdorf }}-Q_{W r} \text {.Neustadt }
\end{aligned}
$$




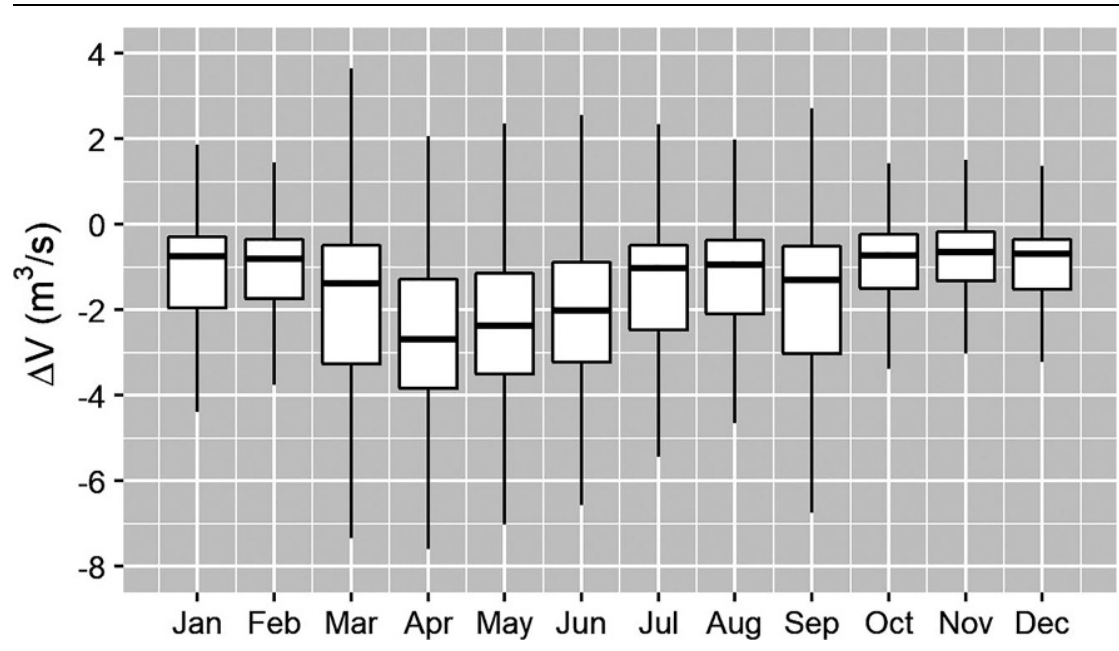

Abb. 2 Versickerung entlang der Leitha im Abschnitt 1 (1979-2014)

Tab. 1 Varianten der im Modell implementierten Versickerung

\begin{tabular}{|c|c|c|c|}
\hline Variante & $\begin{array}{l}\text { Abschnitt } 1 \\
(\%)\end{array}$ & $\begin{array}{l}\text { Abschnitt } 2 \\
(\%)\end{array}$ & Bemerkung \\
\hline 1 & 25,0 & 65,0 & - \\
\hline 2 & 25,0 & 32,5 & - \\
\hline 3 & 25,0 & 65,0 & Speicher in Abs. 2 \\
\hline 4 & 12,5 & 32,5 & - \\
\hline 5 & 25,0 & 32,5 & Speicher in Abs. 2 \\
\hline 6 & 25,0 & 65,0 & Sy konstant 0,1 \\
\hline
\end{tabular}

\section{Methodik}

Für die mathematische Beschreibung von Grundwasserströmungen steht eine Vielzahl an Modellen und Programmen zur Verfügung (Zippel et al. 2010). Einige der verfügbaren Programme sind proprietär und erfordern für deren Nutzung eine Zahlung von Lizenzgebühren. Wird die Erstellung eines Grundwassermodells in Auftrag gegeben, müssen somit Auftraggeber und Auftragnehmer über eine gültige Lizenz verfügen, um Einsicht in alle Projektunterlagen zu bekommen.

\subsection{Verwendete Software}

Neben proprietären Softwarelösungen stehen auch Open-source-Produkte zur Verfügung. Deren Quellcode ist öffentlich für alle Benutzer einsehbar und frei verfügbar. Ein solches Programm stellt das vom United States Geological Survey (USGS) entwickelte MODFLOW (Harbaugh 2005) dar, welches für die gegenständliche Untersuchung herangezogen wurde. Der Nachteil von MODFLOW ist, dass es keine grafische Benutzeroberfläche besitzt und so für die Modellerstellung eine zusätzliche Software benötigt wird. Für die dauer aufgrund des engen Rasters wurde in Kauf genommen, um die kleinräumigen Zuflussflächen bestmöglich abzubilden. Die vertikale Begrenzung des Modells stellen der Grundwasserstauer und die Geländeoberfläche dar. Im Modell wurde ein Layer implementiert, da bei der Auswertung von Bohrprofilen keine zusammenhängenden Schichten unterschiedlicher Durchlässigkeiten gefunden werden konnten.

Neben der räumlichen bedarf es auch einer zeitlichen Diskretisierung des Modells. Die Grundwassergleichung wird für jeden Zeitschritt (Stress Period) mit den gegebenen Randbedingungen gelöst. Die Wahl der Länge der Zeitschritte sollte in Abstimmung auf die verfügbaren Daten stattfinden. Im konkreten Fall stehen viele Daten zu den Grundwasserständen nur wöchentlich zur Verfügung. Es erscheint nicht sinnvoll, die zeitliche Auflösung kleiner als die verfügbaren Eingangsdaten zu wählen. Die Analyse der vorhandenen Daten führten schließlich $\mathrm{zu}$ einem Modellzeitraum von 1. Jänner 2012 bis 20. August 2017. Dieser wurde in 293 Stress Periods zu je 7 Tagen geteilt.

\subsection{Leitha - Grundwasserneubildung durch Infiltration}

Untersuchung wurde das erst kürzlich veröffentlichte QGis (QGIS 2018)-Plugin FREEWAT (Rosetto et al. 2018) verwendet. Damit können Modelldateien in einer Struktur erstellt werden, welche anschließend von MODFLOW eingelesen und verarbeitet werden können. Durch die Implementierung in QGis stehen die gewohnten Werkzeuge für die Bearbeitung von räumlichen Daten zur Verfügung. Diese stellen eine gute Grundlage für die Modellerstellung dar. Alle Daten werden zentral in Form von Tabellen in einer SQLite-Datenbank gespeichert, um einen einfachen Datenaustausch zwischen Projektpartnern zu gewährleisten. Das FREEWAT-Plugin stellt derzeit noch keine ausreichenden Funktionen zur Bearbeitung von Zeitreihen zur Verfügung. Aus diesem Grund wurden alle Daten mit Zeitbezug mit der freien Statistiksoftware $\mathrm{R}$ (R Core Team 2018) aufbereitet und in die Datenbank übertragen.

\subsection{Diskretisierung}

Um ein Modell numerisch zu lösen, muss das Modellgebiet räumlich diskretisiert werden. Für die Diskretisierung wurde ein regelmäßiges Raster von $20 \times 20$ m gewählt. Die längere Rechen-
Wie im Kap. 2. beschrieben, hat die Leitha einen maßgeblichen Einfluss auf den Grundwasserhaushalt im Untersuchungsgebiet. Der Durchfluss im Modellzeitraum war bekannt und die Versickerung wurde durch sechs Varianten abgeschätzt. Der Zufluss ins Grundwasser wurde letztendlich durch eine Brunnenreihe in das Modell implementiert. Die berechnete Versickerungsrate wurde auf die Länge des Flussabschnitts in den jeweiligen Zellen aufgeteilt und für jeden Zeitschritt berechnet.

\subsection{Wasserrechte im Untersuchungsgebiet}

Viele der vergebenen Wasserrechte zur Wasserentnahme im Modellgebiet beziehen sich auf Quell- bzw. Oberflächenwasser und wurden daher nicht näher betrachtet. Neben den Brunnen des Brunnenfeldes Neudörfl wurden noch 12 weitere im Modell implementiert. Alle bewilligten Entnahmen unter $0,11 / \mathrm{s}$ wurden nicht berücksichtigt. Dieser Wert entspricht etwa 0,16\%o der Konsensentnahme der Brunnen im Brunnenfeld Neudörfl. Es wurde 
angenommen, dass dieser Wert unter der Messgenauigkeit der Fördermengen im Brunnenfeld liegt und die Vereinfachung gerechtfertigt ist, diese nicht ins Modell $\mathrm{zu}$ übernehmen. Die Merkur Handelswaren AG besitzt ein Wasserrecht (Postzahl: WN-000008) im Ausmaß von 801/s zur Entnahme von Löschwasser. Da nicht davon ausgegangen wird, dass dieser Brunnen regelmäßig genutzt wird, wurde diese Nutzung im Modell ebenfalls nicht berücksichtigt. Die Wasserwerke Wiener Neustadt sind im Besitz eines Wasserrechts (Postzahl: WN-001188) für das Brunnenfeld Süd im Umfang von 3601/s. Nach Rücksprache bei den Wasserwerken wurde mitgeteilt, dass dieses Brunnenfeld zum Zeitpunkt der Modellerstellung noch nicht zur Gänze in Betrieb war. Zwei der fünf errichteten Brunnen förderten zu dem Zeitpunkt gemeinsam etwa $5 \mathrm{l} / \mathrm{s}$.

Außer den bewilligten und im Wasserbuch einsehbaren Entnahmemengen stehen keine Informationen über die tatsächlichen Fördermengen zur
Verfügung. Aus diesem Grund wurden alle Brunnen, außer jene im Brunnenfeld Neudörfl und die der Wasserwerke Wiener Neustadt, konstant mit einer Nutzung im Ausmaß des Konsenses angesetzt. Für die quantitative Verfügbarkeit des Grundwassers kann dieser Zustand als „Worst Case“ angesehen werden. Die berücksichtigten Brunnen und deren Entnahmemengen sind in Tab. 2 ersichtlich.

\subsection{Randzufluss}

Wie bereits beschrieben ist der östliche Modellrand durch das Rosaliengebirge begrenzt. Der Westhang der Erhebung entwässert in das südliche Wiener Becken und wird daher auch im Modell berücksichtigt. Vor allem infiltrierter Niederschlag fließt unterirdisch dem Grundwasserkörper und somit dem Modellgebiet zu. Dieser Umstand wurde als unterirdischer Randzufluss berücksichtigt und als Brunnenreihe im Modell implementiert. Kupfersberger et al. (2014) haben sich schon inten-
Tab. 2 Im Modell berücksichtigte Wasserrechte

\begin{tabular}{|l|l|}
\hline Postzahl & Konsensentnahme $(1 / \mathrm{s})$ \\
\hline WB-000035 & 0,17 \\
\hline WB-003219 & 1,00 \\
\hline WB-003521 & 0,50 \\
\hline WN-000582 & 1,00 \\
\hline WB-000233 & 1,00 \\
\hline WN-000020 & 17,00 \\
\hline B30905 & 0,12 \\
\hline B25716 & 0,46 \\
\hline WN-001111 & 12,50 \\
\hline WN-001188 & 5,00 \\
\hline WB-002554 & 1,40 \\
\hline WN-001135 & 2,00 \\
\hline
\end{tabular}

siv mit den unterirdischen Zuflüssen in den Grundwasserkörper beschäftigt und dafür eigens ein NiederschlagAbfluss-Modell erstellt. Die Ergebnisse wurden in das gegenständliche Modell übernommen. Zwei unterschiedliche Größen des Randzuflusses wurden definiert. Dieser beträgt $8,1 \mathrm{l} / \mathrm{s} / \mathrm{km}$ ober-

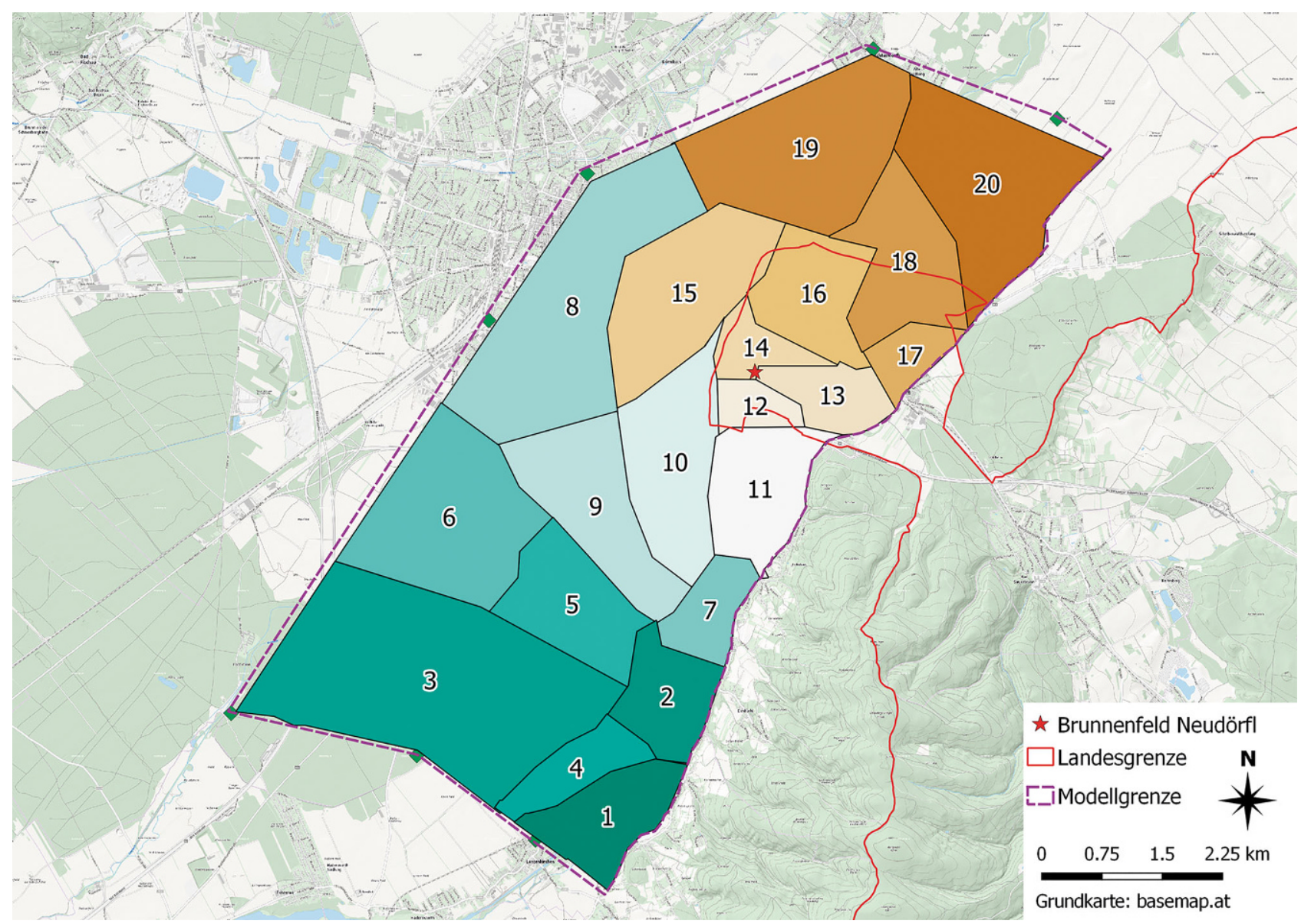

Abb. 3 Zoneneinteilung des Modellgebiets für die Kalibrierung 
halb und 16,11/s/km unterhalb des Katzelsdorfer Rauwehrs.

\subsection{Niederschlag}

Der Niederschlag im Modellgebiet wurde aus dem Mittelwert von drei Messstellen (N109918, N110106, N110411) berechnet. Da jeder Wassertropfen, der auf die Erdoberfläche fällt, noch eine Bodenpassage durchsickern muss, um schlussendlich zur Grundwasserneubildung beizutragen, wurde der Niederschlag über einen virtuellen linearen Speicher gedämpft, um der zeitlichen Verzögerung Rechnung zu tragen. Des Weiteren wurde angenommen, dass nur $80 \%$ des gefallenen Niederschlags infiltrieren.

\subsection{Modellkalibrierung}

Nach der Implementierung der Randbedingungen musste das Modell kalibriert werden. Dabei galt es, die Parameter hydraulische Durchlässigkeit $\left(k_{f}\right)$ und Speicherkoeffizient $\left(S_{y}\right)$ so zu optimieren, dass die gemessenen mit den simulierten Wasserständen möglichst gut übereinstimmen. Im ersten Schritt wurde das Modellgebiet in 20 Zonen geteilt, in denen die Parameter jeweils als konstant angesehen wurden. Bei der Einteilung der Zonen wurde versucht, Regionen mit ähnlichen hydrogeologischen Eigenschaften zusammenzufassen. In Abb. 3 ist die Abgrenzung der Zonen dargestellt. Nach der erfolgten Einteilung wurden alle für die Berechnung in MODFLOW benötigten Input-Daten mit dem FREEWAT-Plugin erzeugt.

FREEWAT bietet die Möglichkeit, eine Modellkalibrierung mittelst UCODE 2014 (Poeter et al. 2014) durchzuführen. Programme zur automatisierten Kalibrierung sind bei systematischer Vorgangsweise bestrebt, einen gewissen Zielwert zu erreichen, beispielsweise ein Minimum der summierten quadrierten Fehler (SSE, sum of squared errors). Die Parameteroptimierung mit UCODE lieferte nach den ersten Modellläufen allerdings keine zufriedenstellenden Ergebnisse. So wurde nach einer alternativen Möglichkeit gesucht, um die Parameter für dieses Modell bestmöglich $\mathrm{zu}$ optimieren. Es wurde entschieden, die Kalibrierung nach einem Ansatz der sogenannten MonteCarlo-Simulation durchzuführen. Bei dieser Methode werden viele Simula-

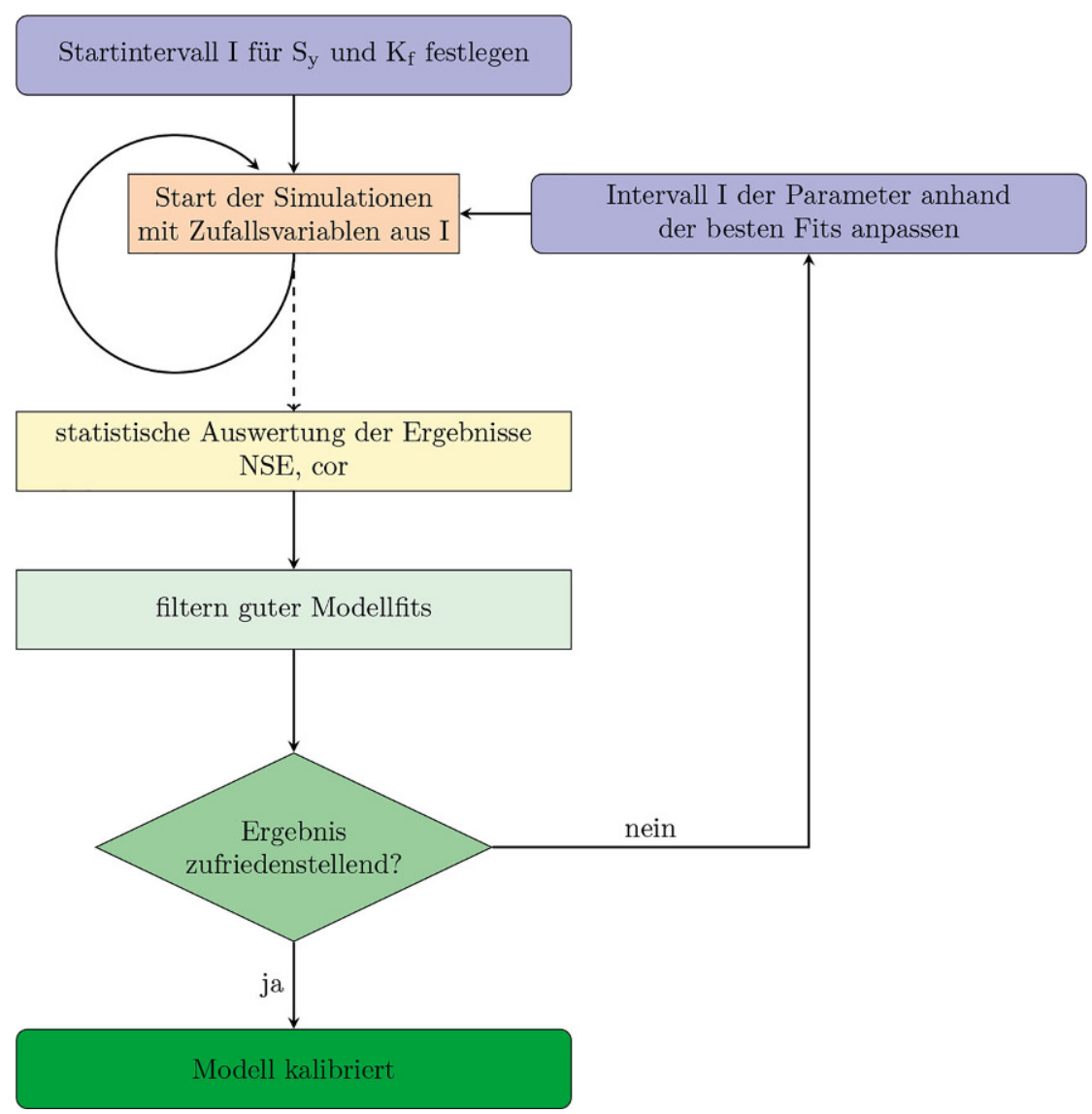

Abb. 4 Vorgehensweise bei der Modellkalibrierung

tionen mit Zufallszahlen durchgeführt und anschließend ausgewertet.

Grundsätzlich kann die verwendete Methode als halbautomatisiert zusammengefasst werden. In Abb. 4 ist der Ablauf der Optimierung dargestellt. Für den ersten Schritt wurde ein Python-Skript geschrieben, welches die Parameter $\mathrm{S}_{\mathrm{y}}$ und $\mathrm{k}_{\mathrm{f}}$ der jeweiligen Zonen ändert. Alle Input-Daten für MODFLOW werden als Textdateien abgespeichert, weshalb diese einfach zu handhaben sind. Die Parameter werden in einer Matrix gespeichert, die der Größe des Modellrasters entspricht. Für die Kalibrierung wurden 20 zufällige Werte aus einem vorgegebenen Intervall für $\mathrm{k}_{\mathrm{f}}$ und ebenso viele für $\mathrm{S}_{\mathrm{y}}$ generiert. Das Erstellen von Zufallsvariablen, das Schreiben dieser in die Input-Datei und das Starten der Simulation wurden im Skript automatisiert und in einer Schleife wiederholt. Nach jeder Simulation wurden die verwendeten Parameter und die damit simulierten Wasserstände in den Grundwassermessstellen abgespeichert. Die Auswertung der Ergebnisse erfolgte in der Regel täglich, um das Intervall anzupassen, aus dem Zufallszahlen generiert wurden. Für die Beurteilung der Modellgüte jeder Simulation wurde die Nash-SutcliffeEffizienz der wichtigsten Messstellen summiert. Dadurch konnte die Bewertung eines Modelllaufs anhand einer einzigen Zahl einfach durchgeführt werden.

Nachdem dieser Prozess einige Male durchlaufen war, konnte das Ergebnis nicht mehr signifikant verbessert werden. Die erhaltenen Resultate wurden als ausreichend genau angesehen. Damit endete der Prozess der Parameteroptimierung für das Modell. Diese Vorgangsweise wurde für alle sechs Varianten parallel durchgeführt. Die so erhaltenen Parametersätze stellen die Grundlage für die weitere Ermittlung der Zuflussflächen dar.

\subsection{Ermittlung der Zuflussfläche im Brunnenfeld Neudörfl}

Das Ergebnis einer MODFLOW-Simulation ist der Wasserstand im Modellgebiet zu jedem Zeitschritt, und in jeder 
aktiven Zelle. Die Grundwasserströmung folgt dem Gradienten der Grundwasseroberfläche. Somit lässt sich auf der Grundlage dieses Ergebnisses die Filtergeschwindigkeit ermitteln. Diese ist nach Darcy abhängig von der hydraulischen Durchlässigkeit und der Neigung der Grundwasseroberfläche. Die Filtergeschwindigkeit stellt jedoch keine reale Größe dar. Erst durch die Bildung des Quotienten aus Filtergeschwindigkeit und effektiver Porosität kann die mittlere Fließgeschwindigkeit (Abstandsgeschwindigkeit) berechnet werden. Da es für die effektive Porosität keine zuverlässige Datengrundlage gibt, musste diese abgeschätzt werden. In der gegenständlichen Untersuchung wurde der kalibrierte Wert für $\mathrm{S}_{\mathrm{y}}$ als effektive Porosität herangezogen.

Die Entnahmemenge im Brunnenfeld beeinflusst die Größe der Zuflussfläche maßgeblich. Deshalb wurden jene Zeiträume näher untersucht, in denen besonders hohe Entnahmen aus dem Grundwasserkörper stattfanden. Da angedacht war, auch die Variabilität der Zuflussflächen zu ermitteln, wurden auch Zeiträume minimaler Entnahmen identifiziert. Dabei wurden nicht die Tagesfördermengen, sondern die Maxima und Minima des gleitenden Mittels über einen Zeitraum von 60 Tagen für die Betrachtung herangezogen. Die ermittelten Zeiträume beziehen sich auf eine maximale beziehungsweise minimale Entnahme aus dem gesamten Brunnenfeld.

Eine weitere Annahme ist, dass nicht nur die entnommenen Wassermengen aus dem Grundwasser die Zuflussflächen beeinflussen, sondern dass auch der überregionale Grundwasserstand einen Einfluss auf die Strömungen im Brunnenfeld hat. Deswegen wurde die Entscheidung getroffen, für die Jahre 2012-2016 jeweils die 60 Tage mit der höchsten und geringsten Entnahme zu untersuchen. Den Unsicherheiten bei der Festlegung eines ungünstigen Systemzustands in Bezug auf die Größe des Einzugsgebiets soll somit Rechnung getragen werden. Eine Gegenüberstellung der Tagesfördermengen mit dem gleitenden Mittel über 60 Tage derselben ist in Abb. 5 dargestellt.

Um nun das Einzugsgebiet des Brunnens in den ausgewählten Zeiträumen zu bestimmen, wurde die Methode des backward particle tracking des Programms MODPATH (Pollock 2012) angewandt. Dabei wurden 400 virtuelle Partikel in den Brunnen gelegt und de-

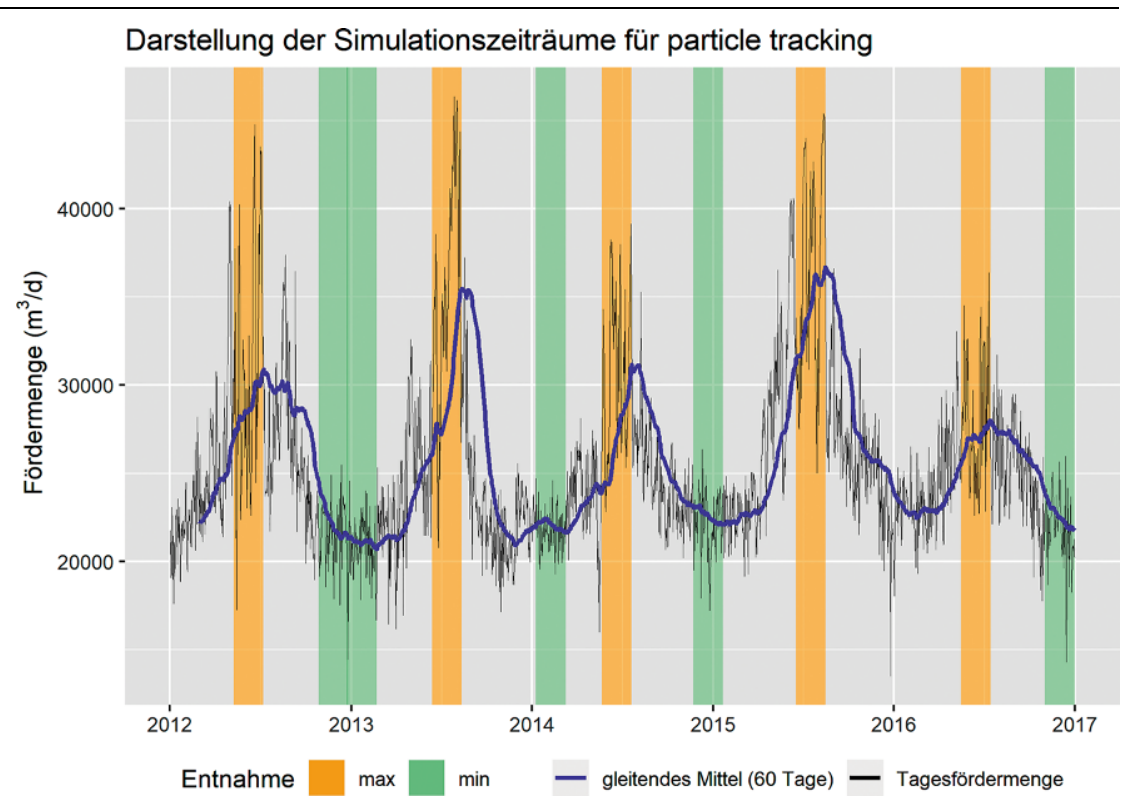

Abb. 5 Tagesfördermenge aus dem Brunnenfeld und Zeiträume, in denen die Zuflussflächen berechnet wurden

ren Bahnlinien von dort ausgehend, rückwärts in der Zeit, ermittelt. Die Koordinaten der Partikel wurden regelmäßig gespeichert und standen zur Auswertung zur Verfügung. Anzumerken ist, dass bei der anschließenden Ermittlung der Zuflussflächen die vertikale Komponente der Partikel nicht berücksichtigt wurde. Diese Vorgangsweise stellt somit einen konservativen Ansatz dar, da die Entnahme aus dem Grundwasserkörper nicht kontinuierlich über die gesamte Tiefe des Brunnens erfolgt.

\subsection{Anzahl der ausgewerteten Parametersätze}

Die vorherrschenden hydrogeologischen Randbedingungen konnten im Zuge der Kalibrierung nicht eindeutig ermittelt werden. Bei der Auswertung der Ergebnisse wurden viele Modellläufe mit ähnlich guter Anpassung an die gemessenen Wasserstände identifiziert. Deshalb wurde entschieden, etwa die besten 50 Ergebnisse jeder Variante weiter auszuwerten. Da sich die Parametersätze untereinander jedoch unterscheiden, konnten durch

Simulation mit zufälligen $\quad$ Auswertung der Ergebnisse $\quad$ Filtern guter Ergebnisse Parametersätzen

\section{für weitere Bearbeitung}
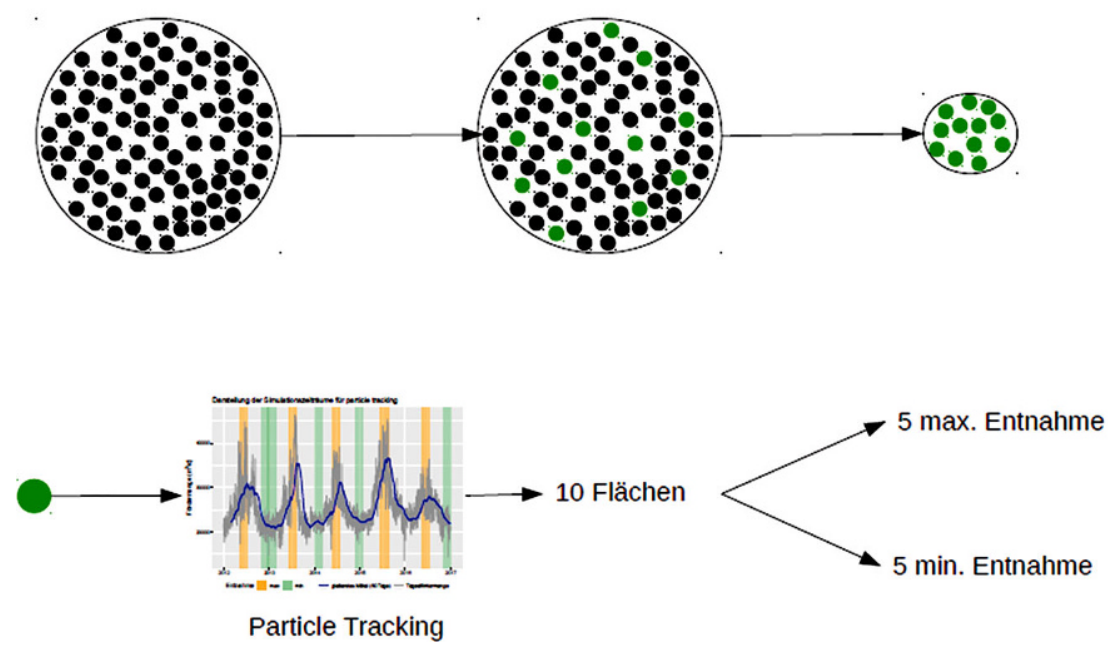

Abb. 6 Anzahl der durchgeführten Berechnungen 
die Auswertung mehrere Modellläufe auch hydrogeologische Unsicherheiten berücksichtigt werden.

Die untere Hälfte der Abb. 6 zeigt die Vorgangsweise der weiteren Analyse. Mit jedem Parametersatz wurde particle tracking in 10 Zeiträumen durchgeführt. Nach der weiteren Auswertung der Daten wurden dadurch 10 Zuflussflächen $\mathrm{zu}$ jedem Brunnen generiert. Diese setzen sich aus den Zeiträumen der minimalen und maximalen Entnahmen in den Jahren 2012-2016 zusammen. Durch die gewählte Vorgangsweise wurden für jeden Brunnen 3020 Zuflussflächen berechnet.

\subsection{Automatisierte Ermittlung der Einzugsgebietsflächen}

Wegen der großen Anzahl an durchgeführten Berechnungen musste eine automatisierte Methode zur Auswertung der Zuflussflächen entwickelt werden. Das Ergebnis des particle trackings stellt eine Textdatei dar, in welcher Koordinaten von Punkten der Bahnlinien gespeichert sind. Für die weiteren Analysen musste aus der erhaltenen Punktwolke ein umrandendes Polygon erzeugt werden. Nicht nur Endpunkte, sondern auch Bahnpunkte können die gesuchte Fläche begrenzen. Aus diesem Grund mussten alle berechneten Punkte der Bahnlinien in die Auswertung einbezogen werden.

Die Möglichkeit, ein umhüllendes Polygon zu definieren, welche für diesen Zweck am geeignetsten erschien, bietet das R-Package alphahull (Pateiro-Lopez und Rodriguez-Casal 2016). Durch die Anwendung der Funktion ahull_track() konnte eine Liste mit Kreisbögen berechnet werden, welche die Punktwolke begrenzen. Da die Kreisbögen in der Liste nicht in der korrekten Reihenfolge abgespeichert waren, mussten die Bögen neu angeordnet werden. Dafür wurde eine Funktion geschrieben, welche jeweils die Abstände der End- und Anfangspunkte eines Bogens mit allen anderen berechnet und vergleicht, um das nächste Segment zu identifizieren und richtig einzureihen. Die Funktion wurde in einem Skript implementiert und in einer Schleife für alle Punktwolken wiederholt. Mit dieser Methode ist es gelungen, die Daten schnell und automatisiert auszuwerten.

\section{Ergebnis}

\subsection{Ermittelte Parameter für das} Untersuchungsgebiet

Im Zuge der Kalibrierung konnten für verschiedene Werte der hydraulischen Durchlässigkeit einer Zone ähnlich gute Simulationsergebnisse erzielt werden. Das mag dadurch bedingt sein, dass die gewählten Parameter der umliegenden Zonen den Wasserstand in der betrachteten Zone beeinflussen. Somit hängt das Ergebnis nicht nur von einem Parameter, sondern von der jeweiligen Kombination dieser Parameter ab. Auch der Umstand, dass während der Kalibrie-
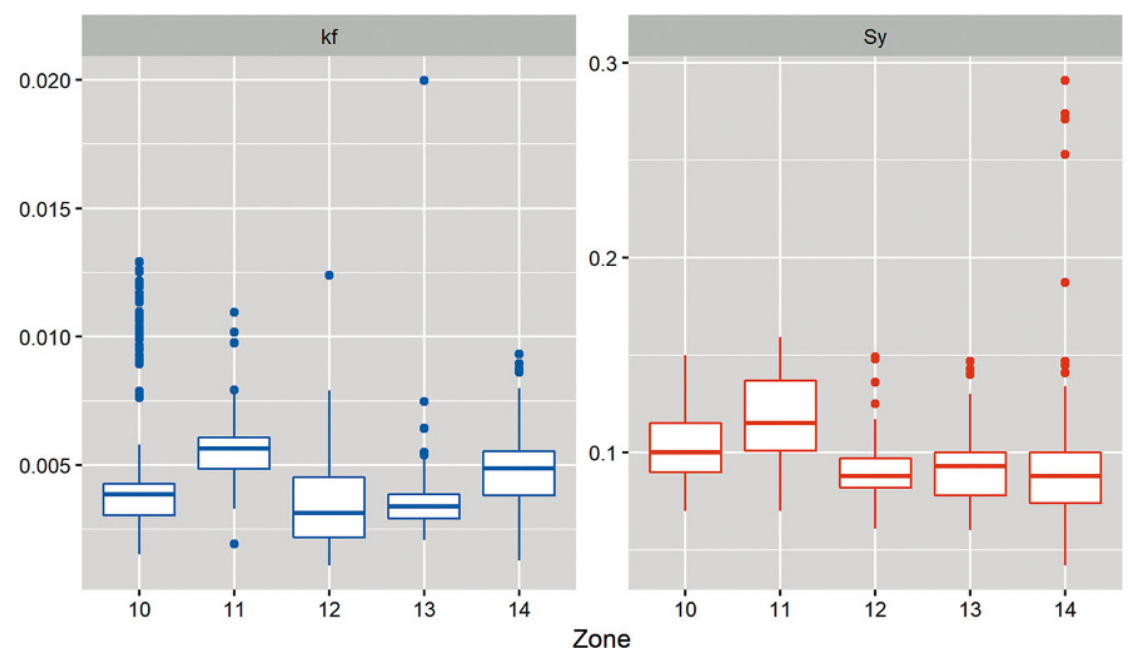

Abb. 7 Kalibrierte Parameter in den Zonen nahe dem Brunnenfeld

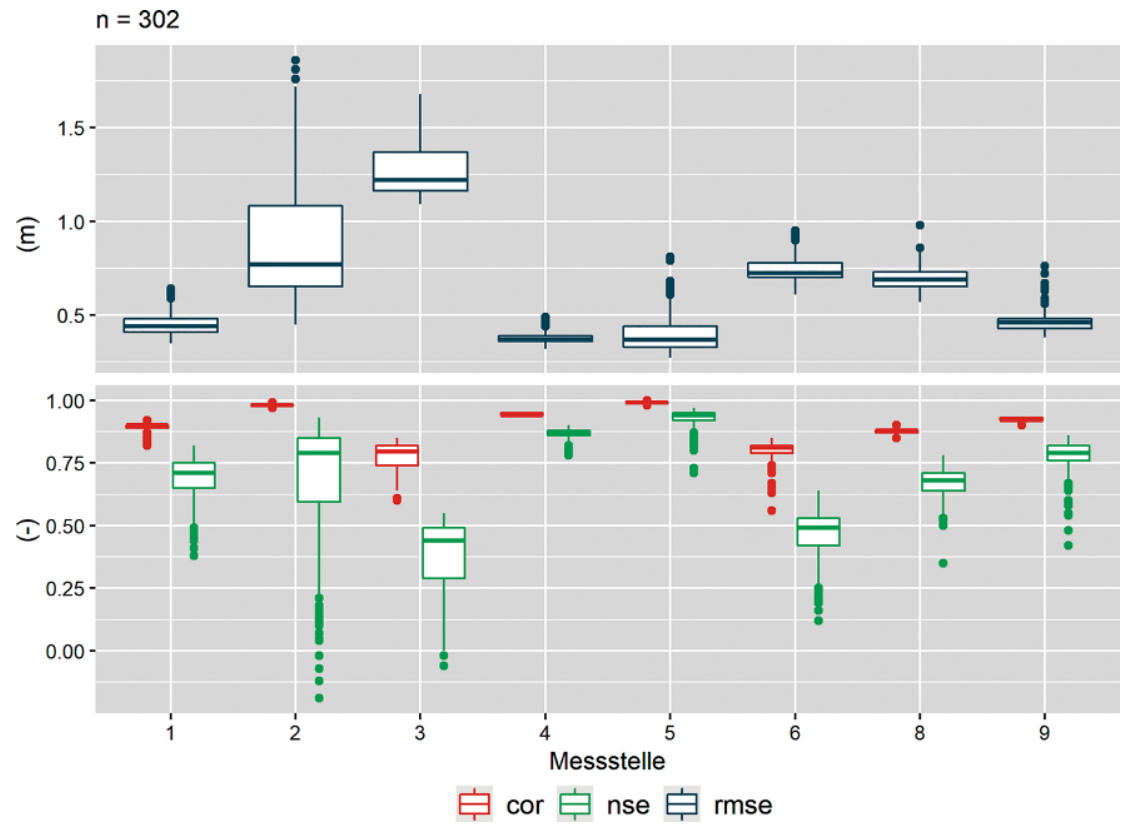

Abb. 8 Modellgüte aller Simulationen 


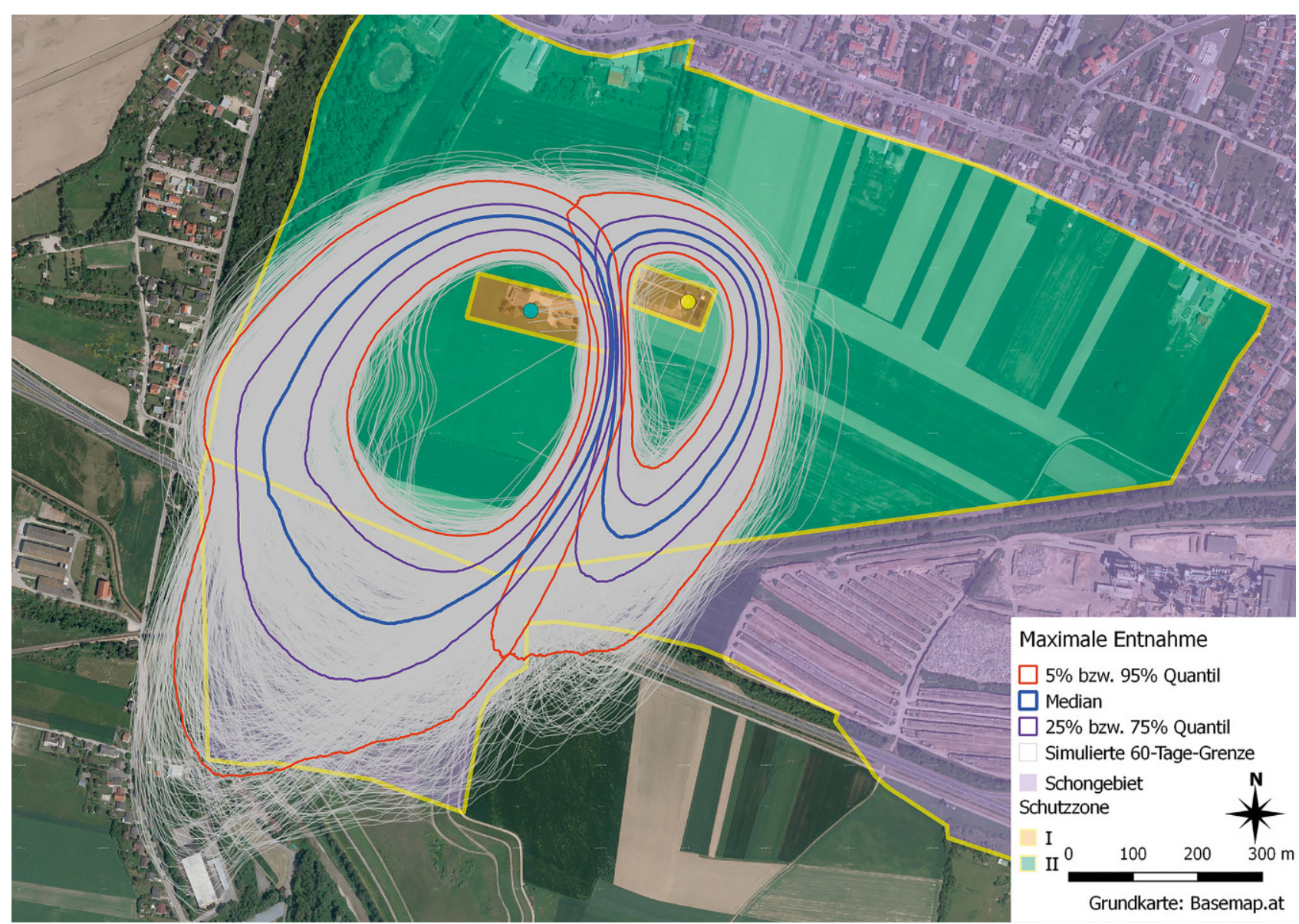

Abb. 9 Ermittelte 60-Tage-Zuflussflächen mit statistischer Auswertung

durchgeführte Studien vorgenommen. In der Untersuchung von Kupfersberger et al. (2014) wurde ein Speicherkoeffizient von 0,1 für das gesamte südliche Wiener Becken angenommen. Nachtnebel und Holzmann (2001) haben in der durchgeführten Modellierung, in der die Leitha die östliche Randbedingung war, für Bereiche nahe dem Untersuchungsgebiet Werte von 0,05-0,15 angesetzt. In dieser Größenordnung befinden sich auch die in der Modellierung verwendeten Koeffizienten, welche in Abb. 7 ersichtlich sind.

Nicht alle Parametersätze liefern ein gleich gutes Ergebnis. Eine statistische Auswertung der Verteilung der Modellgüte ist in Abb. 8 dargestellt. In allen Simulationen konnte der zeitliche Verlauf des Grundwasserstandes sehr gut nachgebildet werden. Die Korrelation der simulierten und beobachteten Wasserstände ist in jeder Messstelle im Median nicht schlechter als 0,75. Ein differenzierteres Bild erhält man, wenn die Auswertung der Nash-Sutcliffe-Effizienz (NSE) herangezogen wird. In der gegenständlichen Untersuchung konnten für die meisten Zeitreihen ebenfalls sehr gute Ergebnisse erzielt werden. Der Median der Grundwassermessstelle 2 zeigt eine gute Anpassung vieler Modellläufe, jedoch gibt es Ausreißer mit Werten kleiner 0. Diese kommen zustande, weil bei einigen wenigen Parametersätzen der simulierte Grundwasserstand parallel zum beobachteten verläuft. Das erklärt die durchwegs hohe Korrelation und die Ausreißer, sowohl in Bezug auf die Nash-SutcliffeEffizienz als auch auf den RMSE. Auch Messstelle 3 konnte oft nicht gut abgebildet werden. Angemerkt sei hier, dass sich diese nahe der Leitha befindet und maßgeblich von der unsicheren Randbedingung der Infiltration durch das Flussbett beeinflusst ist. Nicht dargestellt sind die Ergebnisse der Messstelle 7. Der Verlauf dieser Messstelle konnte nicht nachgebildet werden, da sie am Rand des Grundwasserkörpers und in einiger Entfernung zum Untersuchungsgebiet liegt. Daher wurde sie bei der Auswertung nicht weiter berücksichtigt.

\subsection{Analyse der simulierten 60-Tage-} Grenze

Für jeden Zeitraum, in dem particle tracking durchgeführt wurde, konnte ein Polygon generiert werden, welches die Zuflussfläche zum Brunnen innerhalb von 60 Tagen abgrenzt. Im nächsten Schritt wurden die Größen der Polygone statistisch ausgewertet. Um die Entfernung zum Brunnen zu ermitteln, wurden insgesamt 4668 Linien sternförmig, mit Zentrum im jeweiligen Brunnen, angeordnet, um deren Schnittpunkte mit den Polygonen zu ermitteln. So konnte für jede dieser Linien eine statistische Verteilung (Quantile, Median) der Schnittpunkte entlang jeder Linie berechnet werden. Die ermittelten Punkte wurden verbunden und weitere Polygone erzeugt, um die Variabilität der Zuflussflächen grafisch darzustellen. 
Die Ergebnisse der Auswertung für die Zeiträume, bei denen eine maximale Entnahme aus dem Grundwasserkörper stattgefunden hat, sind in Abb. 9 dargestellt. Im Hintergrund sind die Schutz- und Schongebiete ersichtlich. Die Brunnen befinden sich in den orange gefärbten Flächen, der Schutzzone I. Diese umfasst in der Regel den direkten Fassungsbereich des Brunnens und soll vor unmittelbaren Einträgen in den Brunnen schützen. Angemerkt sei zu dieser Grafik, dass pro Brunnen jeweils 1510 Linien der 60-Tage-Zuflussgrenze dargestellt sind. Diese setzen sich aus 302 Modellläufen mit unterschiedlichen
Parametersätzen zu je 5 Zeiträumen zusammen, in denen eine maximale Entnahme aus dem Grundwasserkörper stattgefunden hat. In den Ergebnissen gibt es ein paar Modellläufe, für die die automatisierte Auswertung der Daten nicht perfekt funktionierte. Aus diesem Grund gibt es einzelne Linien, die geradlinig entweder zu den Brunnen oder durch die Zuflussflächen verlaufen. Die weitere Auswertung der Mediane und Quantile konnte so angepasst werden, dass diese Fehler bereinigt wurden. Es bleibt somit lediglich ein optischer Makel in der Abbildung der simulierten Zuflussflächen.
Funding Open access funding provided by University of Natural Resources and Life Sciences Vienna (BOKU).

Open Access Dieser Artikel wird unter der Creative Commons Namensnennung 4.0 International Lizenz (http:// creativecommons.org/licenses/by/4. 0/deed.de) veröffentlicht, welche die Nutzung, Vervielfältigung, Bearbeitung, Verbreitung und Wiedergabe in jeglichem Medium und Format erlaubt, sofern Sie den/die ursprünglichen Autor(en) und die Quelle ordnungsgemäß nennen, einen Link zur Creative Commons Lizenz beifügen und angeben, ob Änderungen vorgenommen wurden.

\section{Literatur}

Harbaugh, A. W. (2005): MODFLOW-2005, The US Geological Survey modular groundwater model: The groundwater flow process. US Department of the Interior.

Kupfersberger, H., Rock, G., Poltnig, W., Reszler, C., Draxler, J. C. (2014): Grundwassermodel südliches Wiener Becken. Technischer Bericht, Johanneum Research.

Nachtnebel, H. P. und Holzmann, H. (2001): Untersuchung der Abflussverhältnisse an der Leitha bei Nieder- und Mittelwasserführung. Endbericht an den Auftraggeber. Im Auftrag der Bgld. u. NÖ Landesregierung und des BMfLFUW. Technischer Bericht, Universität für Bodenkultur Wien, 196 Seiten.

Pateiro-Lopez, B. und Rodriguez-Casal, A (2016): Alphahull, R-Package Version 2.1, URL https://cran.r-project.org/web/packages/alpha hull/index.html, Abruf: 09.05.2019.

Poeter, E. P., Hill, M. C., Lu, D., Tiedeman, C. R. Mehl, S. (2014): UCODE_2014, with new capabi- lities to define parameters unique to predictions, calculate weights using simulated values, estimate parameters with SVD, evaluate uncertainty with MCMC, and More: Integrated Groundwater Modeling Center Report Number: GWMI 2014-02.

Pollock, D.W., (2012): User guide for MODPATH version 6-A particle-tracking model for MODFLOW: U.S. Geological Survey Techniques and Methods, book 6, chap. A41, 58 p.

QGIS (2018): QGis development Team. Qgis geographic information systen. Open source geospatial foundation project. URL: https:// qgis.org/.

R Core Team (2018): R Alanguage and Environment for Statistical Computing. R Foundation for Statistical Computing, Vienna, Austria. URL: https://ww.R-project.org/.

Rosetto, R., DE Filippis, G., Borsi, I., Foglia,

L., Cannata, M., Criollo, R., Vázquez-Suné, E. (2018): Integrating free and open source tools and distributed modelling codes in GIS environment for data-based groundwater management, Environmental Modelling \& Software, 107:210-230.

Zippel, M., Hannapel, S., Duscher, K., Scheytt,

T., Müiller, B. (2010): Mathematische Simulation des Eintrage von Arzneimitteln aus Oberflächengewässern in das Grundwasser durch Uferfiltration. Umweltbundesamt (D), Texte 14/2010. $33-49$.

Hinweis des Verlags Der Verlag bleibt in Hinblick auf geografische Zuordnungen und Gebietsbezeichnungen in veröffentlichten Karten und Institutsadressen neutral. 